\title{
Determination of the NOAA-20 VIIRS TEB RVS from emissive radiation measurements during the Pitch Maneuver
}

\author{
Aisheng Wu $\mathbf{u}^{1}$, Xiaoxiong Xiong ${ }^{2}$ and Kwofu Chiang ${ }^{1}$ \\ ${ }^{1}$ Science and Systems and Applications, Inc., Lanham, MD 20706 \\ ${ }^{2}$ Science and Exploration Directorate, NASA/GSFC, Greenbelt, MD 20771
}

\begin{abstract}
The Visible Infrared Imaging Radiometer Suite (VIIRS) is a key sensor carried on the newly launched (November 18, 2017) Joint Polar Satellite System-1 (JPSS-1), now transitioned to NOAA-20, and the Suomi National Polar-orbiting Partnership (SNPP) satellite. The two VIIRS sensors are nearly identical in design. Its on-board calibration components include a solar diffuser (SD) and a solar diffuser stability monitor (SDSM) for the reflective solar bands (RSB), a V-groove blackbody for the thermal emissive bands (TEB), and a space view (SV) port for background subtraction. These on-board calibrators are located at fixed scan angles. The VIIRS response versus scan angle (RVS) was characterized prelaunch in lab ambient conditions and is currently used to calibrate the on-orbit response for all scan angles relative to the calibrator's scan angle. A spacecraft level pitch maneuver was scheduled during the initial intensive Cal/Val testing for both the NOAA-20 and SNPP. The pitch maneuver provided a rare opportunity for VIIRS to make observations of deep space over the entire scan angle range, which can be used to characterize the TEB RVS. This study provides our analysis of the NOAA-20 pitch maneuver data and assessment of the derived TEB RVS. A comparison between the RVS determined by the pitch maneuver observations and prelaunch lab measurements is conducted for each band, detector, and mirror side of the half angle mirror.
\end{abstract}

Keywords: VIIRS, calibration, response versus scan angle, pitch maneuver, prelaunch

\section{INTRODUCTION}

The VIIRS is a key instrument carried on the newly launched (November 18, 2017) JPSS-1 satellite, now renamed as NOAA-20. This is the second VIIRS instrument operated in space following the first one currently flying on the SNPP satellite ${ }^{1-3}$. The VIIRS sensor design is based on heritage instruments including AVHRR (Advanced Very High Resolution Radiometer), OLS (Operational Linescan System), MODIS (Moderate Resolution Imaging Spectroradiometer), and SeaWiFS (Sea-viewing Wide Field-of-view). It uses a constant-rate rotating telescope assembly 
(RTA) and a double sided half angle mirror (HAM) rotating at half the speed of the RTA. VIIRS has 22 bands with a spectral range from 0.4 to $12.0 \mu \mathrm{m}$ and 7 of them are the thermal emissive bands (TEB). The specified central wavelengths (CW) and bandwidths (BW) for VIIRS TEB are listed in Table 1. The Earth view (EV) swath in the cross-track direction covers a distance of $\sim 3000$ $\mathrm{km}$ over a scan angle range of $\pm 56.0^{\circ}$ off the instrument nadir. Observations by VIIRS cover the entire Earth's surface every one or two days and provide high quality environmental and climate information for land, ocean, and atmosphere. A broad range of Environmental Data Records (EDR) are routinely generated from the VIIRS Sensor Data Records (SDR), which include the calibrated (and geo-located) top of atmosphere (TOA) reflectances, radiances, and brightness temperatures. Like its predecessor, the MODIS instruments on Terra and Aqua, the VIIRS instrument has the calibration capability to monitor its on-orbit performance and to assure data quality over its entire mission.

Table 1. List of VIIRS TEB central wavelengths (CW) and bandwidths (BW) (units in nm)

\begin{tabular}{|c|c|c|c|c|c|c|c|}
\hline Band & M12 & M13 & M14 & M15 & M16 & I04 & I05 \\
\hline CW & 3700 & 4050 & 8550 & 10763 & 12013 & 3740 & 11450 \\
\hline BW & 180 & 155 & 300 & 1000 & 950 & 380 & 1900 \\
\hline
\end{tabular}

The VIIRS on-board calibrators (OBCs) include an SD and an SDSM for the RSB calibration, a V-groove blackbody for the TEB calibration, and a space view (SV) port used to remove the background signals from both RSB and TEB raw responses. The SD and blackbody provide absolute calibration with standards traceable to those provided by the National Institute of Standards and Technology (NIST). Since both the SD and blackbody are located at fixed angles of incidence (AOIs), the calibration at other AOIs is dependent upon the RVS that describes relative response changes to those obtained at the on-board calibrator AOIs. The VIIRS RSB/TEB RVS was characterized prelaunch in lab ambient conditions ${ }^{4,5}$. For TEB, these tests were conducted using the Laboratory Ambient Black Body (LABB) as the target at a number of different scan angles to ensure a good description of the RVS over the entire Earth view scan angle range and space view angle. As expected, the quality of the RVS characterization is vitally important to ensure the quality of the calibrated radiance product. A recent study by Moyer et al.$^{6}$ provided details of the prelaunch RVS characterization, including a comparison of the derived RVS curves between NOAA-20 and SNPP.

During the first several months after launch of NOAA-20, similar to SNPP, there were a number of scheduled spacecraft activities in support of calibration and validation $(\mathrm{Cal} / \mathrm{Val})$ related topics for VIIRS. These calibration maneuvers and their applications were developed based experience and lessons from MODIS ${ }^{7-9}$. The VIIRS pitch maneuver is one of these activities, 
scheduled on January 31, 2018. The purpose of the maneuver is to conduct on-orbit verification of the RVS for VIIRS TEB that was derived from the prelaunch tests ${ }^{4,5}$. Figure 1 gives a snapshot of two Earth view (EV) images at the beginning and near the end of the pitch, respectively. During a normal orbit, the nadir side of the satellite always faces the earth, which is achieved by a continuous pitching of the spacecraft in the forward direction. During the pitch maneuver, however, the spacecraft reversed its pitching direction, which resulted in the spacecraft having a complete view of deep space in the night side of orbit for VIIRS for about 25 minutes. The approach used to derive the RVS from the pitch is based on the measured relative change in the emissive radiation as a function of $\mathrm{AOI}^{10}$. Since the radiance from cold space is nearly zero, the remaining radiance received by the detectors is from the thermal emission of the VIIRS fore optics, including the RTA and HAM. Analysis of detector responses during the pitch maneuver can determine the shape of the TEB RVS. Our previous study ${ }^{10}$ provided a detailed description of the algorithm to derive the RVS for SNPP VIIRS. The current study uses the same algorithm for the newly launched NOAA-20 VIIRS.

Section 2 of this study provides a brief description of the algorithm used to derive the NOAA20 VIIRS TEB RVS. In section 3, the derived results are compared with those determined from the prelaunch tests to check the agreement between the two independent measurements, including detector to detector differences in RVS. A further comparison of the agreement between those for NOAA-20 and SNPP is also included.

\section{METHODOLOGY}

As discussed in our previous study for SNPP VIIRS, to determine the TEB RVS under conditions of the pitch maneuver, it is necessary to make the following assumptions. First, the RVS is determined by measuring the relative change in emissive radiation, which is proportional to the change in the relative response. Second, the RVS is only dependent on AOI, and different VIIRS scan angles may have the same AOI. The RVS is determined using detector responses obtained at two separate sources: deep space and the onboard blackbody. Since the same approach is used for SNPP VIIRS ${ }^{10}$, a detailed procedure to derive the RVS is not included here. At a given EV scan angle, the $R V S_{\mathrm{EV}}$ can be derived:

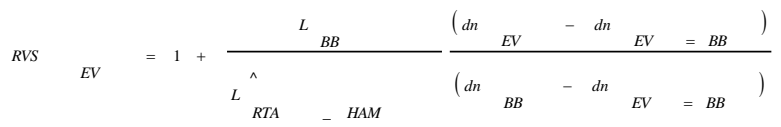

where the $L_{\mathrm{BB}}$ is the radiance of the on-board black body (BB) and 


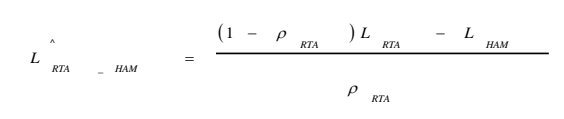

where $L_{\mathrm{RTA}}$ and $L_{\mathrm{HAM}}$ are the radiances from the RTA and HAM, respectively, $\rho_{\mathrm{RTA}}$ is the reflectance of RTA, $d n_{\mathrm{EV}}$ is the background subtracted digital count at the EV scan angle, and $d n_{\mathrm{EV}=\mathrm{BB}}$ is the $d n_{\mathrm{EV}}$ at the scan angle of the $\mathrm{BB}$ view.

A second order polynomial is used to fit the RVS versus AOI, or

$$
\text { RVS } \quad E V \quad=a_{0}+a_{1} A O I+a_{2}{ }_{A O I}^{2}
$$

Coefficients $a_{0}, a_{1}$ and $a_{2}$ are band, detector, and HAM side dependent. Once the coefficients $a_{0}$, $a_{1}$, and $a_{2}$ are produced by the fitting, equation (3) is used to compute the RVS at any given AOI. The VIIRS EV AOI range is from $28.6^{\circ}$ to $56.5^{\circ}$. For on-orbit applications, the RVS is generally normalized to the AOI of the onboard calibrator. For the RSB, the normalization point is the SD $\mathrm{AOI}$ at $61.01^{\circ}$; for the TEB, the RVS is normalized to the BB AOI at $38.53^{\circ}$.

\section{DATA SELECTION}

As shown in Figure 1, the NOAA-20 pitch maneuver started at 13:42:23 GMT and ended at 14:12:15 GMT. Since a complete view of deep space starting from time 13:44:50 GMT, there are nearly 30 minutes of continuous scans available for the RVS analysis. However, before 13:44:50 GMT when there is a complete view of deep space, the cold focal plane temperatures started to rise gradually (Table 2). This is because the sensor's passive radiative cooler is affected by the thermal radiation from the earth's surface during the pitch maneuver. As a result, our data collection is selected from 13:46:41 GMT over a short period of time to avoid any possible impact due to the rapid rise in the cold focal plane temperatures, which started from 13:51:00 GMT, as shown in Figure 2. 

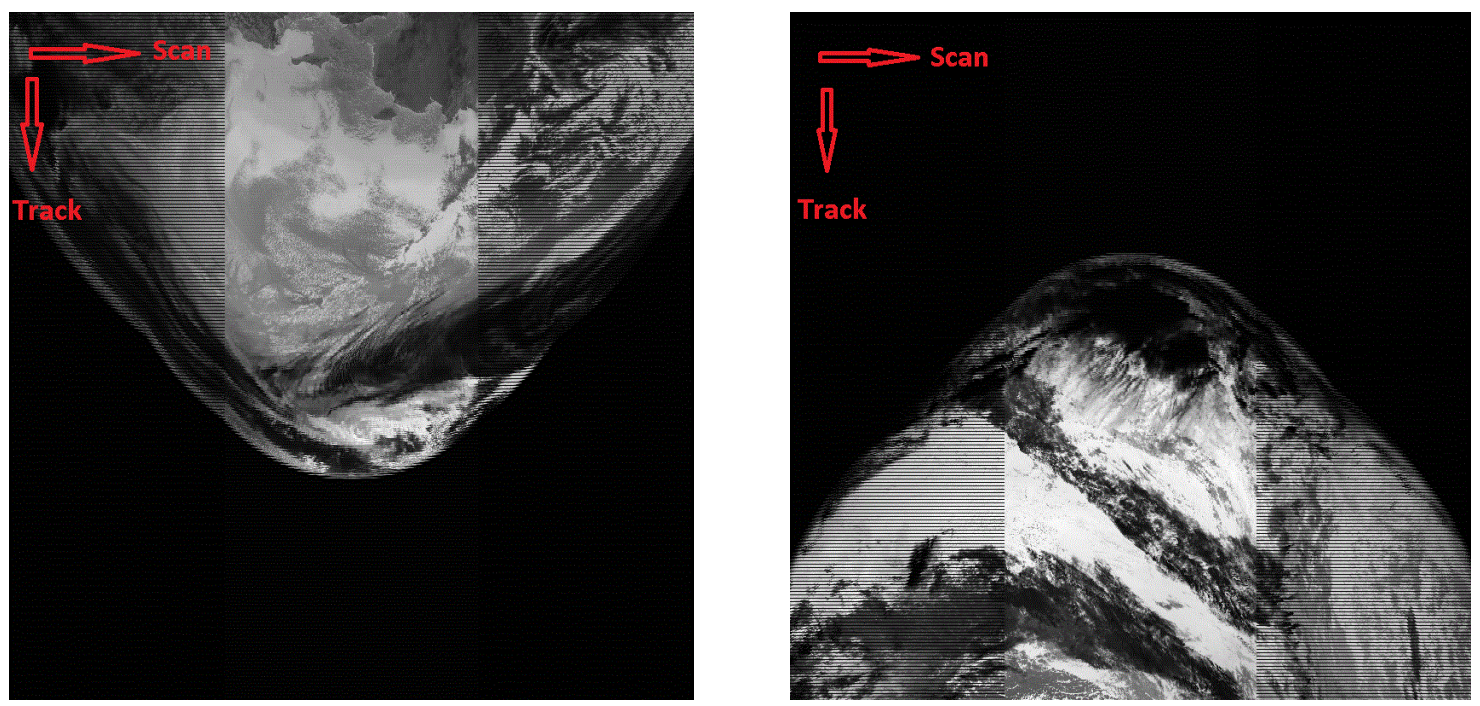

Figure 1. M12 image at the beginning (left) and near the end (right) of the pitch maneuver on January 31,2018 . The entire pitch maneuver period was from 13:42:23 GMT to 14:12:15 GMT. The left image was taken using scans collected from GMT 13:42:23 to 13:46:39 and the right image from GMT 14:07:59 to 14:12:15.

Table 2. A zoom-in list of the focal plane temperatures around data collection

\begin{tabular}{|c|c|c|}
\hline Time (GMT) & S/MWIR (K) & LWIR (K) \\
\hline $13: 40: 58$ & 80.235 & 80.292 \\
\hline $13: 42: 24$ & 80.235 & 80.292 \\
\hline $13: 43: 50$ & 80.238 & 80.294 \\
\hline $13: 45: 16$ & 80.254 & 80.309 \\
\hline $13: 46: 41^{*}$ & 80.289 & 80.341 \\
\hline $13: 48: 05$ & 80.350 & 80.404 \\
\hline
\end{tabular}

* Data collection start time 


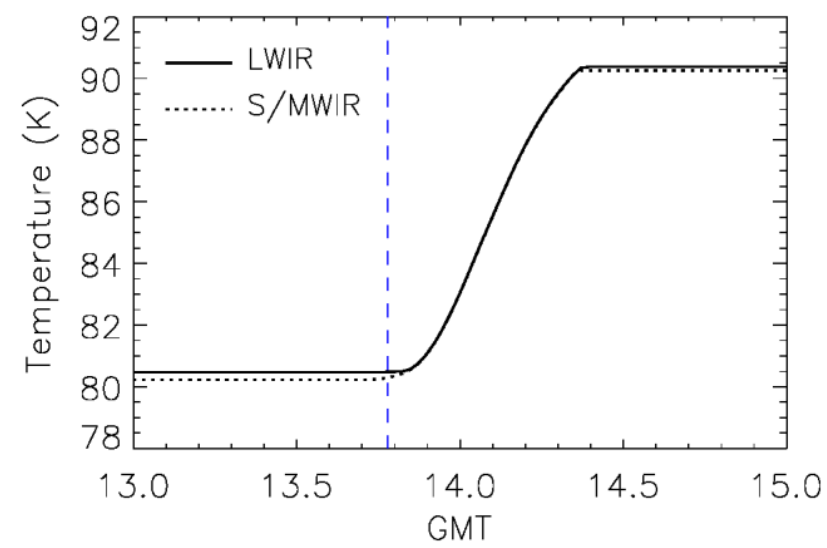

Figure 2. Variations of cold focal plane (S/MWIR and LWIR) temperatures throughout the course of the pitch maneuver. The vertical line is at 13:46:41 GMT when the scan-by-scan data is collected.

A total of 10 consecutive scans ( 5 for each HAM side) of data samples are collected from the pitch maneuver, which are right before a rapid rise in the cold focal plane temperatures. Each scan covers the entire earth view with 3200 pixels for M bands (exception for M13 with 6304 pixels) and 6400 pixels for I bands. Tests with up to 40 scans show that there is little difference in the derived RVS due to high uniformity of space view environment. Figure 3 plots the background subtracted response, dn, versus AOI obtained from one scan of the middle detector, HAM-A side, for M12, M14, M16 and I05. The conversion from the sample number to AOI is based on characteristic information of the sensor design provided the instrument vendor ${ }^{4}$. The background signal can be taken either from the response obtained at the space view port or from the earth view sector obtained at a particular AOI. In this study, we use an average of the last ten samples of the earth view as the background. The fact that the response is not flat (i.e., M14 and I05) across the entire earth view range indicates that the reflectivity of the HAM changes with the scan angle. 

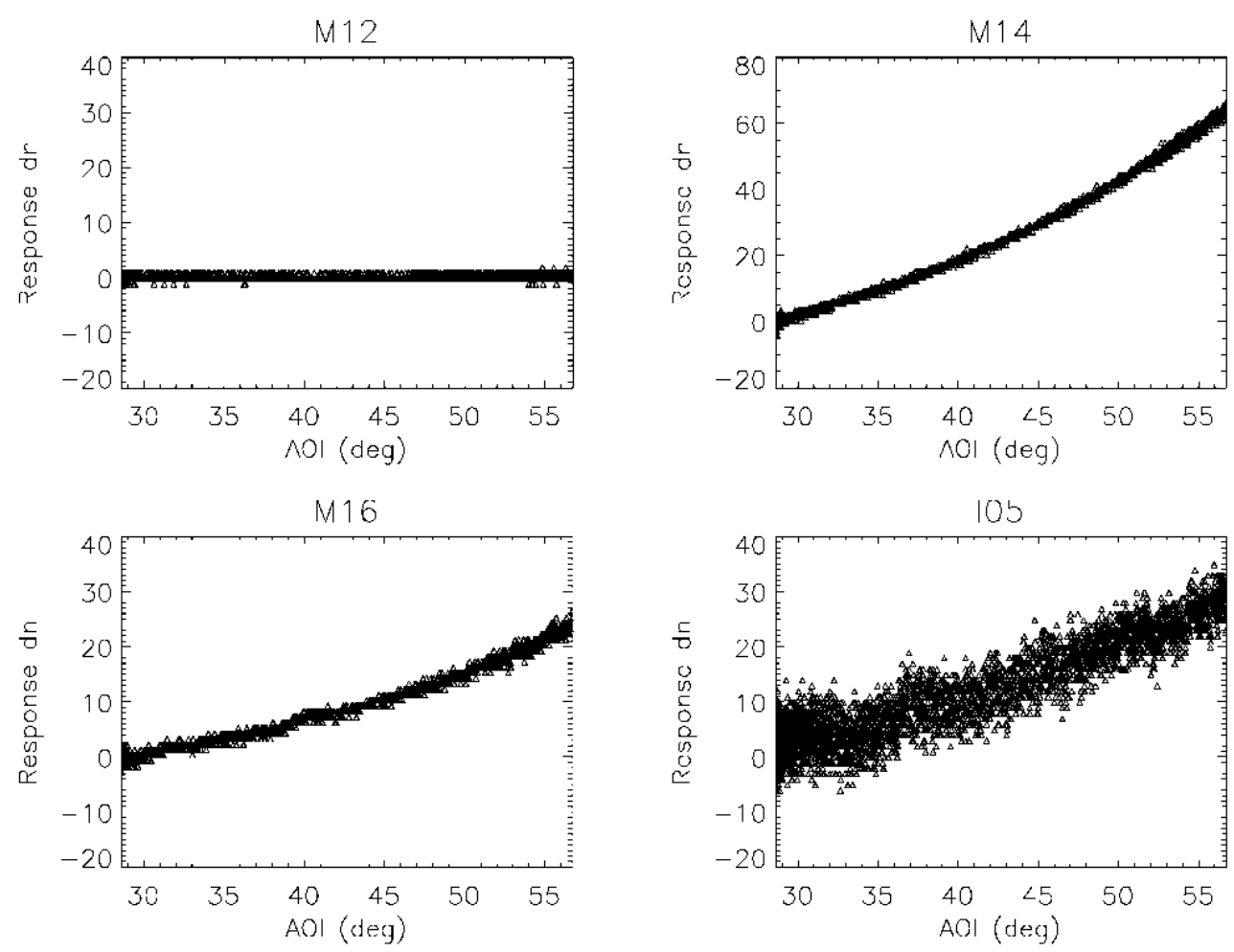

Figure 3. One scan of pixel-level background subtracted response, dn, versus AOI from the middle detector of M12, M14, M16 and I05.

\section{RESULTS AND DISCUSSION}

With the background subtracted response, $d n_{\mathrm{EV}}$, the RVS can be determined using equation (1). Examination of all scans of the pitch maneuver data indicates that the temperatures of the two cold focal planes (S/MWIR and LWIR) has already started to increase before there is a complete deep space view at all AOIs of the EV sector. The rate of the temperature increase is relatively slow ( $\sim 0.026 \mathrm{~K}$ per minute) at the beginning. Thus, it is still not necessary to correct the possible impacts due to cold focal plane temperature variations on the detector response between the beginning and end of each scan, which is less than 1 second. To confirm there is minimal impact of the increase in the cold focal plane temperatures, Figure 4 plots the relative RVS change with the LWIR cold focal plane temperature for M14 and I04. Impact on other bands is much smaller than these two bands. 

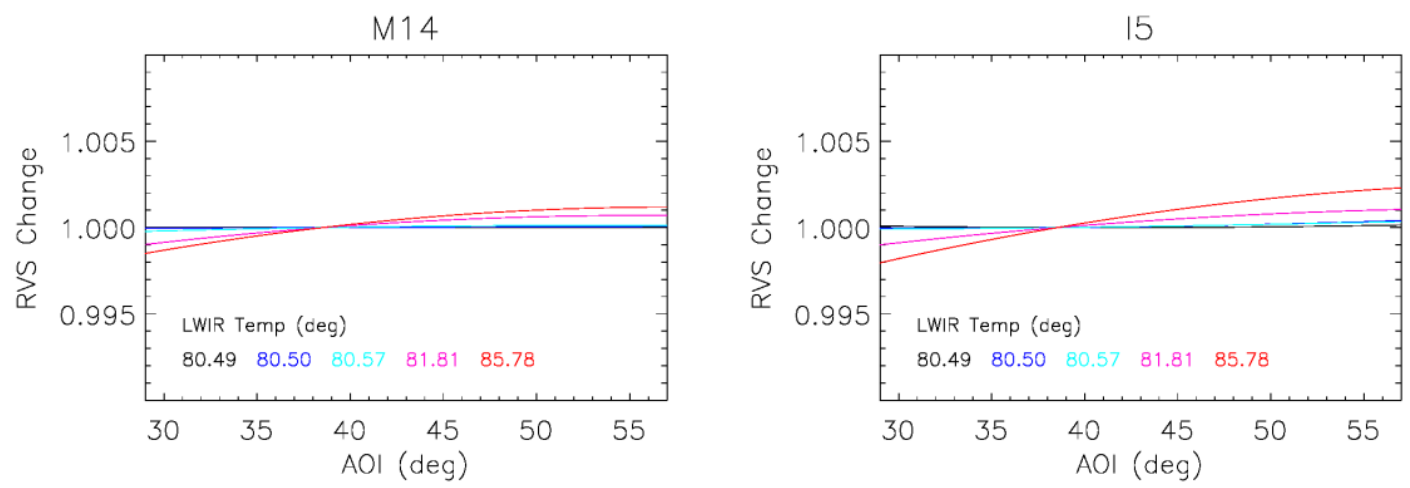

Figure 4. RVS changes at five LWIR focal plane temperatures $(80.49,80.50,80.57,81.81$ and $85.78^{\circ}$ ) relative to the result obtained at $80.40^{\circ}$ for M14 and $\mathrm{I} 05$.

Values of $\rho_{\text {RTA }}$ are taken from the auxiliary prelaunch calibration data provided by the instrument vendor. There are two thermistors used to report RTA temperatures: 1) mainframe bulkhead temperature and 2) mainframe -X scan cavity panel PZ temperature. An average of the two RTA temperatures is used. In addition to the direct use of the averaged RTA temperature, a temperature offset is added based on the uncertainty assessments described in the instrument thermal calibration model ${ }^{11}$. Various values of the offset were examined during the prelaunch tests. Currently, the suggested offset is $-4.0 \mathrm{~K}$. Based on results of analyzing the similar pitch maneuver data from SNPP VIIRS ${ }^{10}$, the temperature offsets have a negligible impact on the RVS as only the relative values are derived.

The RVS determined from Equation (1) is fit versus AOI by a second order polynomial, using Equation (3). The fitting uncertainties (one sigma) are within $0.04 \%$ for all bands except for I04, which shows a slightly higher uncertainty of $0.08 \%$. Figure 5 plots the HAM-A RVS of NOAA-20 VIIRS TEB as a function of AOI. The RVS from prelaunch tests, used in current sensor data record (SDR) lookup tables (LUT), is also plotted in the figure. The LUT RVS is based on prelaunch data collected under sensor ambient conditions ${ }^{6}$. Comparisons of the pitch maneuver and prelaunch RVS show good agreement of within $0.3 \%$ according the ratio of the two RVS (Figure 6). This is also within the target of $0.3 \%$ specified for the characterization of the prelaunch $\mathrm{RVS}^{12}$. Similar agreement is found for both HAM sides. To further examine the consistency of the pitch maneuver based RVS, similar results from SNPP are plotted together with those from NOAA-20 for bands M12, M14, M16 and I05. The results from the two independent assessments are very close, although the current results have slightly better agreement with the prelaunch tests.

It should be noted that the results shown in Figures 5 and 6 are band and HAM-side dependent. The RVS from the pitch maneuver are first derived for individual detectors, except for a few edge detectors. This is because samples at the beginning and end of the scan are filled with 
default value of 65534 for the edge detectors 1-2 and 15-16 of M bands and detectors 1-4 and 2932 of I bands resulting from the bow-tie deletion. Therefore, the RVS derived from the pitch maneuver cannot cover all detectors. The RVS from the pitch maneuver shown in Figures 5 and 6 is the average over most of the detectors without the bow-tie deletion. The RVS obtained from prelaunch measurements is detector dependent and up to $0.2 \%$ differences are observed between the beginning and end of the detector array. By normalizing the detector dependent RVS with the band average, we can examine the detector-to-detector spreads and compare them with the prelaunch based results. Figure 7 shows comparison among three detectors at the beginning, middle and end of the detector array, i.e., detectors 3, 8, and 14 for $\mathrm{M}$ bands and 5, 16, 28 for I bands. Except for the results of M13 which shows up to $0.3 \%$ differences in the pitch data, compared with differences less than $0.1 \%$ in the prelaunch results, results from the other bands are comparable in terms of the detector to detector differences.
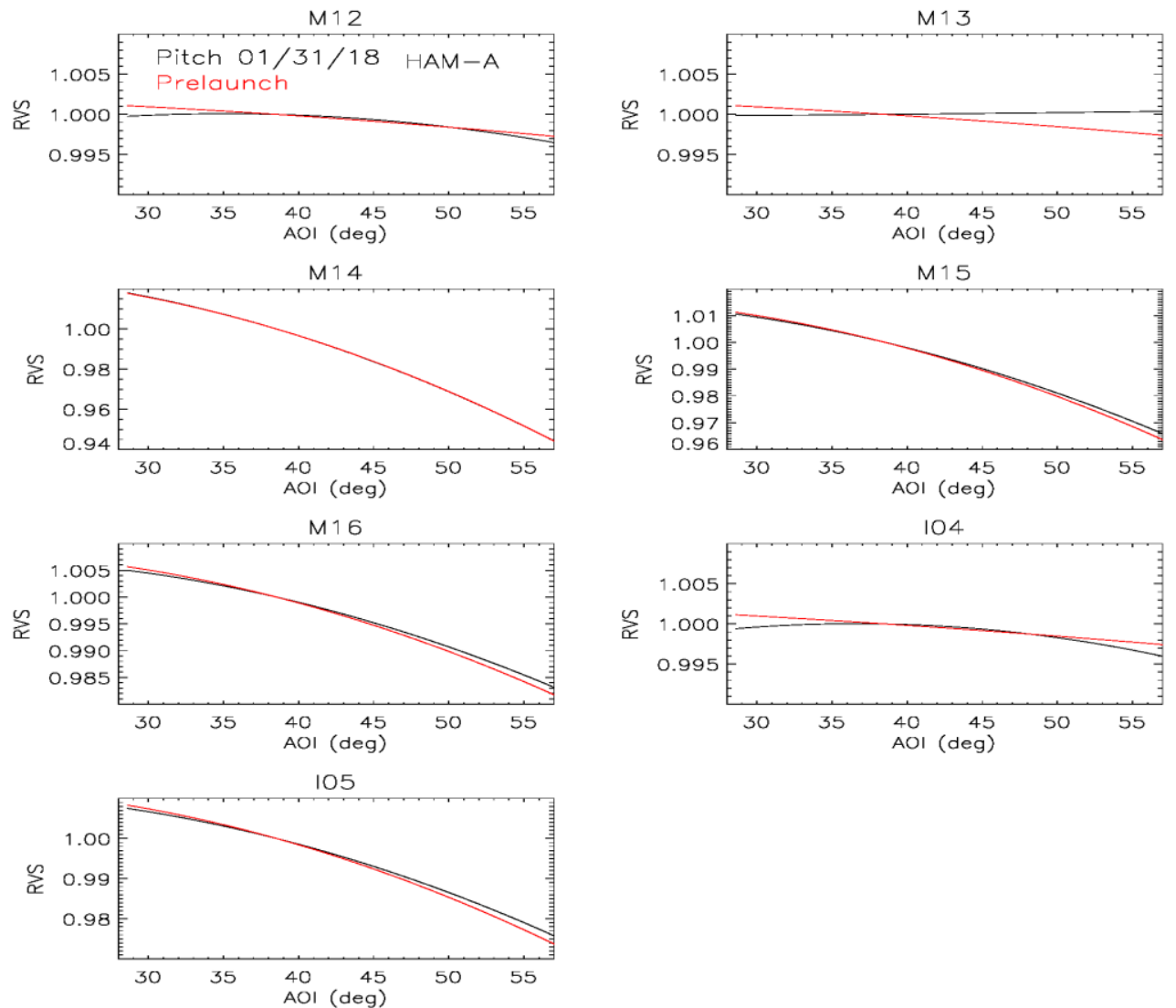

Figure 5. RVS of HAM-A derived from pitch maneuver data (black curves). The curves are determined from Equation 3 after an average over scans and detectors. Also shown is the RVS from the prelaunch (red curves). 

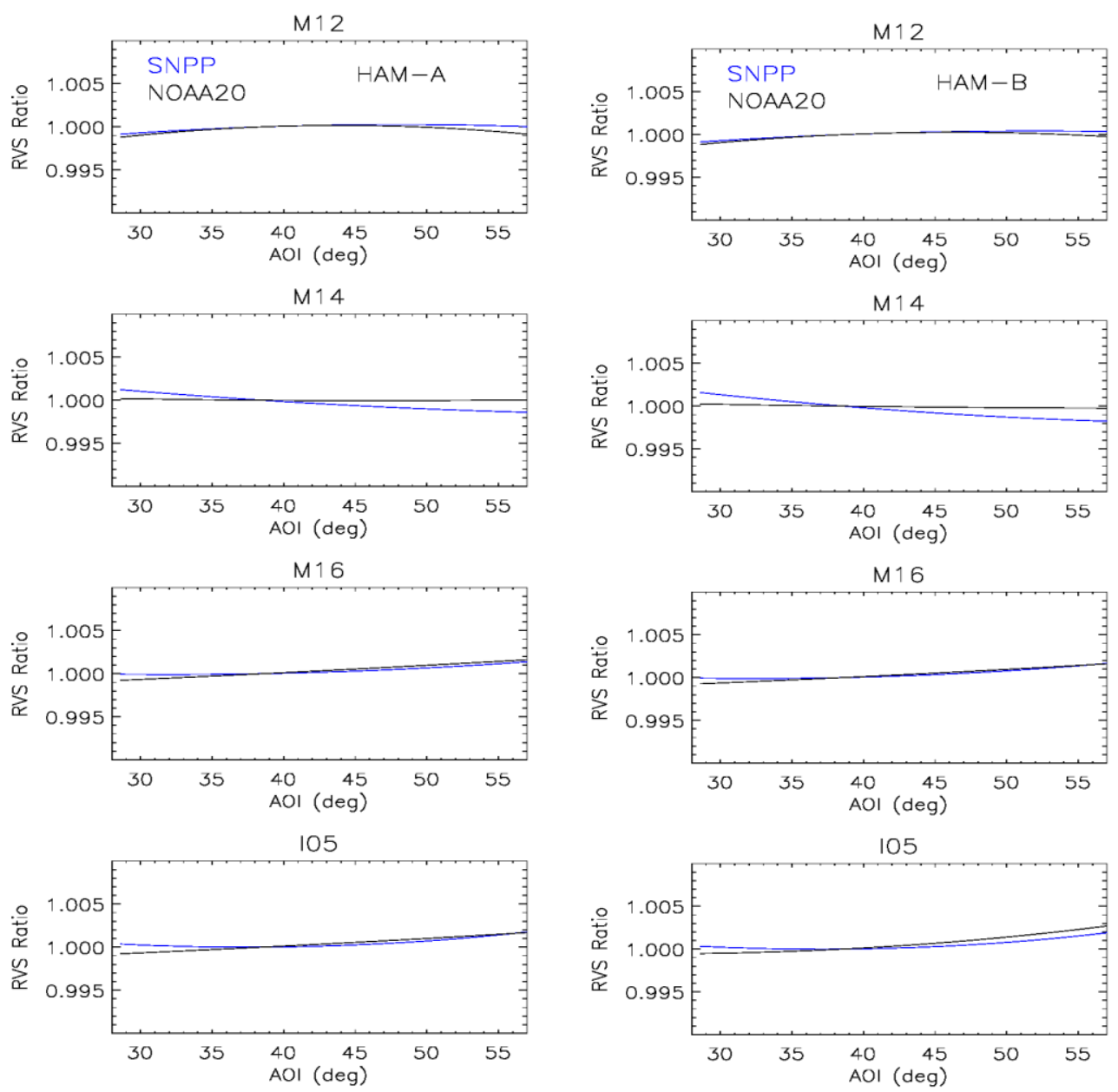

Figure 6. Comparison of pitch-to-prelaunch RVS ratio between SNPP and NOAA-20 for both HAM sides (M12, M14, M16 and I05). 

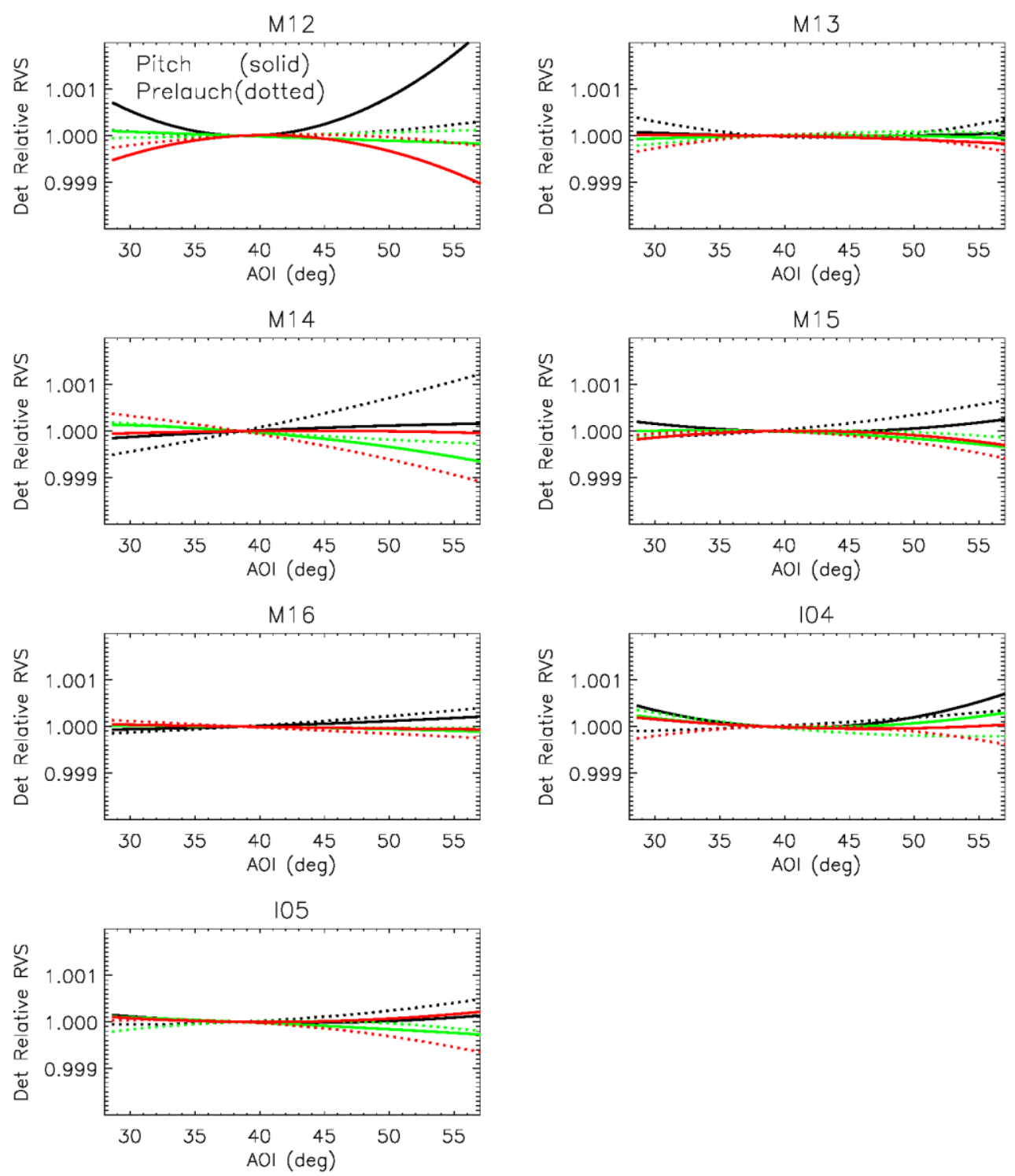

Figure 7. Comparison of detector-to-detector RVS difference between results from the pitch (solid lies) and prelaunch (dotted lines) for NOAA-20. Three detectors at the beginning, middle and end of the detector array are shown here.

In this study, the approach used to derive the RVS is based on the measured relative change in the emissive radiation as a function of scan angle during the pitch maneuver. Since the radiance from cold space is nearly zero, the remaining radiance received by the detectors is from the thermal emission of the VIIRS fore optics, including the RTA and HAM. Analysis of detector responses can determine the shape of the TEB RVS and two independent results from NOAA-20 and SNPP 
pitch maneuvers are in close agreement with those derived from the prelaunch tests. For bands with little change $(<0.3 \%)$ in the RVS magnitude such as M12, M13 and I04, however, there is concern that any small systematic errors in the measured data could cause a bias in the derived RVS.

\section{SUMMARY}

The NOAA-20 maneuver, conducted on January 31, 2018, provided a good opportunity to examine the VIIRS TEB RVS immediately after the launch of the instrument. This study provided a brief description of the methodology applied for on-orbit assessment of the RVS and an illustration of the RVS results derived using data collected from the sensor's deep space view during a spacecraft pitch maneuver. The results are compared with those derived from prelaunch tests. Results show that the pitch maneuver based RVS agrees well with the prelaunch values to within $0.3 \%$. Such differences are likely due to the fact that the pitch-based method relies on the measured relative change in emissive radiation, while the prelaunch-based method relies on a lab controlled blackbody source. The results of this study have clearly demonstrated that the prelaunch TEB RVS, used in current SDR LUT, is well characterized.

\section{ACKNOWLEDGMENTS}

Authors would like to acknowledge the technical assistance provided by Jeff McIntire from NASA VIIRS Characterization Support Team (VCST).

\section{REFERENCES}

1. "National Polar-orbiting Operational Environmental Satellite Systems (NPOESS) Preparatory Project (NPP) Instrument Calibration and Support Element Design Document," NASA/GSFC, January 10, 2008, unpublished.

2. Cao, C., X. Xiong, S. Blonski, Q. Liu, S. Uprety, X. Shao, Y. Bai, and F. Weng, Suomi NPP VIIRS sensor data record verification, validation, and long-term performance monitoring. J. Geophys. Res. dio: 10.1002/2013JD020418, 2013.

3. Xiong, X., J. Butler, K. Chiang, B. Efremova, J. Fulbright, N. Lei, J. McIntire, H. Oudrari, J. Sun, Z. Wang, and $\mathrm{A}$. Wu, VIIRS on-orbit calibration methodology and performance. J. Geophys. Res. Atmos., 119, 50655078, 2014.

4. "Response vs. Scan Angle (FP-10) Test Procedure for VIIRS (TP154640-250)", Raytheon, May 24, 2007.

5. Wu, A., J. Mclntire, X. Xiong, F. J. De Luccia, H. Oudrari, D. Moyer, S. Xiong, and C. Pan, "Comparison of VIIRS Prelaunch RVS Among Independent Studies", Proceedings of SPIE - Earth Observing Systems XVI, Vol. 8153, DOI: 10.1117/12.892468, 2011. 
6. Moyer, D., J. McIntire, H. Oudrari, J. McCarthy, X. Xiong, and F. De Luccia, “JPSS-1 VIIRS Pre-Launch Response Versus Scan Angle Testing and Performance”, Remote Sens., 8(2), 141; doi:10.3390/rs8020141, 2016.

7. Xiong, X., Vincent V. Salomonson, K. Chiang, A. Wu, B. Guenther, and W. Barnes, "On-orbit characterization of RVS for MODIS thermal emissive bands", Proc. SPIE 5652, doi: 10.1117/12.578344, 2004.

8. Xiong, X., J. Sun, J. Esposito, X. Liu, W. L. Barnes, and B. Guenther, "On-orbit characterization of a solar diffuser's bidirectional reflectance factor using spacecraft maneuvers", Proc. SPIE 5151, doi: 10.1117/12.504802, 2003.

9. Butler, J., X. Xiong, R. Barnes, F. Patt, J. Sun, and K. Chiang, "An overview of Suomi NPP VIIRS calibration maneuvers", Proc. SPIE 8510, doi: 10.1117/12.930993, 2012.

10. Wu, A., X. Xiong, and K. Chiang, Postlaunch assessment of the response versus scan angle for the thermal emissive bands of visible infrared imaging radiometer suite on-board the Suomi national polar-orbiting partnership satellite, Journal of Applied Remote Sensing, 11(4), 044002, 2017.

11. Joint Polar Satellite System (JPSS) Visible Infrared Radiometer Suite (VIIRS) Sensor Data Records (SDR) Algorithm Theoretical Basis Document (ATBD); Technical Report; Goddard Space Flight Center: Greenbelt, MD, USA, 2013.

12. "VIIRS Radiometric Calibration Algorithm Theoretical Basis Document ATBD (ref Y3261)", Northrop Grumman Aerospace Systems, Document Number: D43777, Revision: F, 2010. 